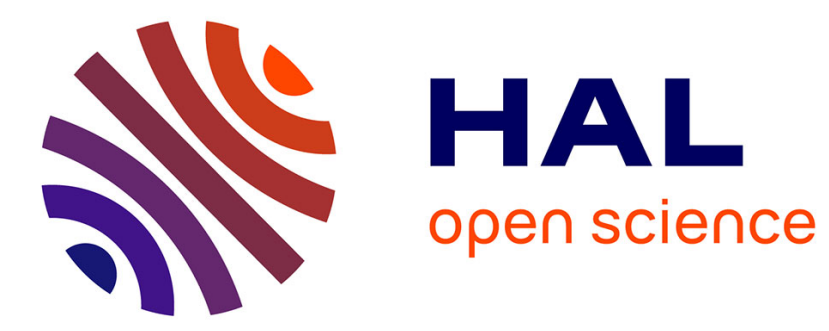

\title{
Méthode de prévision des équilibres vibratoires dans les systèmes couplés par utilisation des valeurs propres et des vecteurs propres de couplage
}

\author{
F. Bessac, L. Gagliardini, Jean-Louis Guyader
}

\section{- To cite this version:}

F. Bessac, L. Gagliardini, Jean-Louis Guyader. Méthode de prévision des équilibres vibratoires dans les systèmes couplés par utilisation des valeurs propres et des vecteurs propres de couplage. Journal de Physique IV Proceedings, 1994, 04 (C5), pp.C5-761-C5-764. 10.1051/jp4:19945165 . jpa-00252845

HAL Id: jpa-00252845

https://hal.science/jpa-00252845

Submitted on 1 Jan 1994

HAL is a multi-disciplinary open access archive for the deposit and dissemination of scientific research documents, whether they are published or not. The documents may come from teaching and research institutions in France or abroad, or from public or private research centers.
L'archive ouverte pluridisciplinaire HAL, est destinée au dépôt et à la diffusion de documents scientifiques de niveau recherche, publiés ou non, émanant des établissements d'enseignement et de recherche français ou étrangers, des laboratoires publics ou privés. 


\title{
Méthode de prévision des équilibres vibratoires dans les systèmes couplés par utilisation des valeurs propres et des vecteurs propres de couplage
}

\author{
F. BESSAC, L. GAGLIARDINI et J.-L. GUYADER*
}

Centre Scientifique et Technique du Bâtiment, 24 rue Joseph Fourier, 38400 Saint-Martin-d'Hères, France * Laboratoire Vibrations-Acoustique, INSA Lyon, Bâtiment 303, 20 avenue Albert Einstein, 69621 Villeurbanne cedex, France

\begin{abstract}
Coupled multimodal systems are investigated using a coupling, dimensionless matrix, revealing the change in the vibrations of isolated systems. In the case of two plates coupled by a spring, this matrix presents a single eigenvalue which depends on input mobility. The disturbance of uncoupled systems can then be simply written by projections onto the associated coupling eigenvector. This approach allows us to characterize precisely the coupling and to consider important reductions in computation time for estimating the energy transfer between coupled vibroacoustic systems. This analysis presents a criterion of weak coupling. It also offers interesting perspectives for the understanding of the energetical methods for coupled systems.
\end{abstract}

\section{INTRODUCTION}

La propagation du bruit dans les structures mécaniques et les rayonnements acoustiques associés sont généralement décrits en introduisant la notion de couplage entre différents sous-systèmes (fluide, structures, sous-structures).

Les approches énergétiques (SEA, Power flow) utilisées en moyennes et hautes fréquences, décrivent de manière efficace les échanges par couplage entre différents sous-systèmes à l'aide d'une approche phénoménologique. Ce couplage est alors caractérisé par des facteurs issus d'expériences éventuellement numériques.

Les approches purement numériques au contraire décrivent parfaitement les interactions entre systèmes mais ne proposent pas de modèle pour les phénomènes qui gouvernent les échanges. Pour des raisons de coût, elles sont de plus limitées au domaine des basses fréquences.

L'approche développée ici s'appuie sur les modes des sous-systèmes et se donne comme objectif de caractériser le couplage à l'aide de grandeurs caractéristiques offrant des possibilités d'exploitation variées. Dans le cas simple de deux plaques couplées par un ressort, l'introduction de valeurs propres et vecteurs propres de couplage permet de mettre en évidence la part du couplage dans la solution du problème.

\section{DEUX STRUCTURES COUPLÉES : EQUATION DU COUPLAGE}

Dans le cas de deux structures vibrantes couplées par un ressort, on peut décrire la vitesse $\mathrm{w}_{\mathrm{i}}(\mathrm{x}, \mathrm{y})$ et la force d'excitation $F_{o i}(x, y)$ de la plaque $i(i=1,2)$ sur la base des modes $\psi_{i_{n}}(x, y)$ des sous-systèmes (ref. $[1,2]$ ) de la manière suivante : 


$$
w_{i}(x, y)=\sum_{n} a_{i_{n}} \psi_{i_{n}}(x, y) \quad \text { et } \quad F_{o i}(x, y)=\sum_{n} b_{i_{n}} \psi_{i_{n}}(x, y)
$$

$\{\overrightarrow{\mathrm{w}}\}$ et $\left\{\overrightarrow{\mathrm{F}}_{\mathbf{o}}\right\}$ étant les vecteurs des amplitudes modales $\mathrm{a}_{\mathbf{i}}$ des vitesses et $\mathrm{b}_{\mathbf{i}}$ des forces d'excitation, et $[\mathrm{Z}]$, la matrice d'impédance, la relation d'équilibre des deux structures couplées s'écrit comme suit :

$$
\left[\begin{array}{ll}
\mathrm{Z}_{11} & \mathrm{Z}_{12} \\
\mathrm{Z}_{21} & \mathrm{Z}_{22}
\end{array}\right]\left\{\begin{array}{l}
\overrightarrow{\mathrm{w}}_{1} \\
\overrightarrow{\mathrm{w}}_{2}
\end{array}\right\}=\left\{\begin{array}{l}
\overrightarrow{\mathrm{F}}_{\mathrm{o} 1} \\
\overrightarrow{\mathrm{F}}_{\mathrm{o} 2}
\end{array}\right\}
$$

A partir de cette expression, en divisant chaque ligne par le bloc d'impédance diagonal, on obtient l'équation du couplage :

$$
\{\vec{w}\}-[C]\{\vec{w}\}=\left\{\vec{w}_{o}\right\} \text { et }[C]=\left[\begin{array}{cc}
0 & C_{12} \\
C_{21} & 0
\end{array}\right]
$$

[C] est la matrice de couplage, adimensionnelle, et $\left\{\vec{w}_{0}\right\}$ le vecteur des amplitudes modales des vitesses des systèmes découplés.

Dans ce qui suit, on s'intéressera au problème simple de deux plaques couplées par un ressort de raideur $\mathrm{k}$ : les plaques sont homogènes, élastiques et supposées être simplement appuyées sur leur pourtour (figure 1).

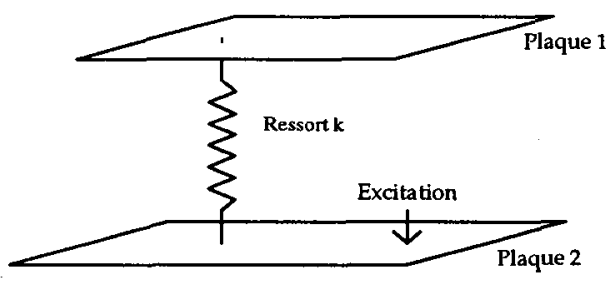

Figure 1 : modèle utilisé

\section{VALEURS PROPRES ET VECTEURS PROPRES DE COUPLAGE}

On montre que la matrice de couplage [C] admet un unique couple de valeurs propres de couplage $\lambda^{ \pm}$, opposées, et donc un unique couple de vecteurs propres. Si $Y_{i}\left(R_{i}\right)$ représente la mobilité de la plaque $i$ au point d'attache du ressort $\left(\mathrm{x}_{\mathrm{R} i}, \mathrm{y}_{\mathrm{Ri}}\right)$ et $\omega$ la pulsation de l'excitation, ces deux valeurs propres ont pour expression analytique :

$$
\lambda^{ \pm}= \pm \frac{k}{j \omega} \sqrt{Y_{1}\left(R_{1}\right) Y_{2}\left(R_{2}\right)}
$$

Il est intéressant de noter que cette valeur propre est accessible expérimentalement.

Comme le montre la figure 2, aux 4 basses fréquences, la valeur propre de couplage est constante. En hautes fréquences, lorsque les deux systèmes se découplent, la valeur propre tend vers zéro. Dans la zone intermédiaire (zone de résonance masse-ressortmasse), la forte interaction entre les deux systèmes se traduit par des valeurs propres de couplage supérieures à 1 .

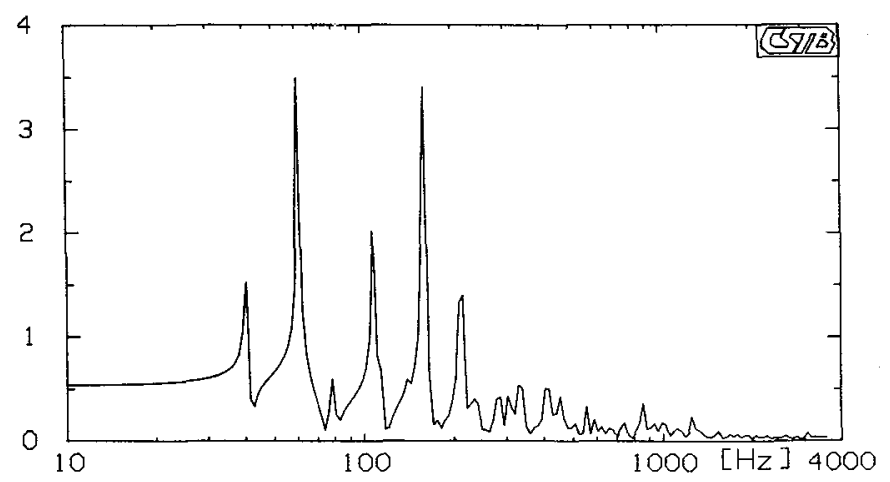

Figure 2 : Module de la valeur propre de couplage pour deux plaques en acier de dimensions $(1,4 \times 1 \times 0,01)$ et $(1,7 \times 1,2 \times 0,02) \mathrm{m}$, un ressort de raideur $k=10^{7} \mathrm{~N} . \mathrm{m}$ situé en $(x=1,1 ; y=0,88)$ m sur chaque plaque. 
La valeur propre de couplage apparaît être représentative de la force de couplage entre les deux systèmes.

Les vecteurs propres, définis à une constante près, sont fonctions des valeurs de la base modale au point de couplage $\Psi_{i_{n}}\left(R_{i}\right)$ et des impédances modales $Z_{i_{n}}$ des systèmes découplés considérés :

$$
\varphi_{\mathrm{m}}^{(1)}=\frac{1}{\sqrt{\mathrm{Y}_{1}\left(\mathrm{R}_{1}\right)}} \frac{\psi_{1_{\mathrm{m}}}\left(\mathrm{R}_{1}\right)}{\mathrm{Z}_{1_{\mathrm{m}}}} \text { et } \varphi_{\mathrm{n}}^{(2)}= \pm \frac{1}{\sqrt{\mathrm{Y}_{2}\left(\mathrm{R}_{2}\right)}} \frac{\psi_{2_{\mathrm{n}}}\left(\mathrm{R}_{2}\right)}{\mathrm{Z}_{2_{\mathrm{n}}}}
$$

Dans ce cas de couplage ponctuel, les vecteurs propres traduisent le comportement résonant des structures excitées au point de couplage. Les vecteurs propres caractérisent les chemins de transmission associés au couplage et peuvent être utilisés pour calculer les déformées issues du couplage, des plaques. L'état vibratoire qui en est déduit, est à rapprocher de la notion de mode hybride (ref. [3]).

La symétrie des valeurs propres et vecteurs propres de couplage permet d'exprimer tout vecteur dans la base d'un des deux vecteurs propres de couplage sous la forme : $\left\{\begin{array}{l}\alpha_{1} \varphi^{(1)} \\ \alpha_{2} \varphi^{(2)}\end{array}\right\}$, où les $\alpha_{i}$ sont les coefficients propres de couplage. La propriété suivante apparaît alors :

$$
\left[\begin{array}{cc}
0 & C_{12} \\
C_{21} & 0
\end{array}\right]\left\{\begin{array}{l}
\alpha_{1} \vec{\varphi}^{(1)} \\
\alpha_{2} \vec{\varphi}^{(2)}
\end{array}\right\}=\lambda\left\{\begin{array}{l}
\alpha_{2} \vec{\varphi}^{(1)} \\
\alpha_{1} \vec{\varphi}^{(2)}
\end{array}\right\}
$$

\section{RECONSTRUCTION DE LA SOLUTION}

Les vecteurs vitesse $\{\vec{w}\}$ et $\left\{\vec{w}_{0}\right\}$ sont décomposés sur la base des vecteurs propres de couplage, et complétés d'un terme résiduel qui représente la partie orthogonale aux vecteurs propres de couplage.

$$
\left\{\begin{array}{c}
\overrightarrow{\mathrm{w}}_{1} \\
\overrightarrow{\mathrm{w}}_{2}
\end{array}\right\}=\left\{\begin{array}{c}
\alpha_{1} \vec{\varphi}^{(1)} \\
\alpha_{2} \vec{\varphi}^{(2)}
\end{array}\right\}+\left\{\begin{array}{l}
\overrightarrow{\mathrm{R}}_{1} \\
\overrightarrow{\mathrm{R}}_{2}
\end{array}\right\} \text { et }\left\{\begin{array}{c}
\overrightarrow{\mathrm{w}}_{\mathrm{o} 1} \\
\overrightarrow{\mathrm{w}}_{\mathrm{o} 2}
\end{array}\right\}=\left\{\begin{array}{c}
\alpha_{\mathrm{o} 1} \vec{\varphi}^{(1)} \\
\alpha_{\mathrm{o} 2} \vec{\varphi}^{(2)}
\end{array}\right\}+\left\{\begin{array}{l}
\overrightarrow{\mathrm{R}}_{\mathrm{o} 1} \\
\overrightarrow{\mathrm{R}}_{\mathrm{o} 2}
\end{array}\right\}
$$

Par définition, le produit de la matrice de couplage [C] avec le terme résiduel est nul. On applique donc la matrice de couplage à l'équation du couplage (2) pour éliminer les termes résiduels :

$$
\left.[C]\left\{\begin{array}{l}
\vec{w}_{1} \\
\vec{w}_{2}
\end{array}\right\}-[C]\left\{\begin{array}{l}
\vec{w}_{1} \\
\vec{w}_{2}
\end{array}\right\}=\left\{\begin{array}{l}
\vec{w}_{o 1} \\
\vec{w}_{o 2}
\end{array}\right\}\right) \Leftrightarrow \lambda\left\{\begin{array}{l}
\alpha_{2} \vec{\varphi}^{(1)} \\
\alpha_{1} \vec{\varphi}^{(2)}
\end{array}\right\}-\lambda^{2}\left\{\begin{array}{l}
\alpha_{1} \vec{\varphi}^{(1)} \\
\alpha_{2} \vec{\varphi}^{(2)}
\end{array}\right\}=\lambda\left\{\begin{array}{l}
\alpha_{o 2} \vec{\varphi}^{(1)} \\
\alpha_{o 1} \vec{\varphi}^{(2)}
\end{array}\right\}
$$

De cette expression, une relation entre les coefficients propres de la vitesse des systèmes couplés $\alpha_{i}$ et les coefficients propres de la vitesse des éléments isolés $\alpha_{\mathrm{oi}}$ est extraite :

$$
\alpha_{1}=\frac{\alpha_{\mathrm{o} 1}+\lambda \alpha_{\mathrm{o} 2}}{1-\lambda^{2}} \text { et } \alpha_{2}=\frac{\alpha_{\mathrm{o} 2}+\lambda \alpha_{\mathrm{o} 1}}{1-\lambda^{2}}
$$

Les coefficients propres $\alpha_{o}^{(i)}$ peuvent être calculés analytiquement et valent :

$$
\alpha_{\mathrm{o} 1}=\frac{\mathrm{w}_{\mathrm{o} 1}\left(\mathrm{R}_{1}\right)}{\sqrt{\mathrm{Y}_{1}\left(\mathrm{R}_{1}\right)}} \text { et } \alpha_{\mathrm{o} 2}=\frac{\mathrm{w}_{\mathrm{o} 2}\left(\mathrm{R}_{2}\right)}{\sqrt{\mathrm{Y}_{2}\left(\mathrm{R}_{2}\right)}}
$$

La construction de la solution de l'équation du couplage (2) est obtenue en utilisant la relation (5). Les coefficients propres étant maintenant connus, cela conduit à l'expression : 


$$
\left\{\begin{array}{l}
\vec{w}_{1} \\
\vec{w}_{2}
\end{array}\right\}=\left\{\begin{array}{l}
\vec{w}_{o 1} \\
\vec{w}_{02}
\end{array}\right\}+\lambda\left(\begin{array}{l}
\alpha_{2} \\
\alpha_{1}
\end{array}\right)\left\{\begin{array}{l}
\vec{\varphi}^{(1)} \\
\vec{\varphi}^{(2)}
\end{array}\right\}
$$

La vitesse des systèmes couplés apparaît comme la somme des vitesses des systèmes découplés et de l'effet du couplage décrit uniquement par les valeurs propres et vecteurs propres de couplage.

\section{GÉNÉRALISATION, SOLUTION ITÉRATIVE DES PROBLÈMES COUPLÉS}

Dans le cas de $\mathrm{N}$ points de couplage, on observe $\mathrm{N}$ couples de valeurs propres. L'examen des valeurs propres qui traduisent la force des couplages, permet de classer les chemins de transmissions et éventuellement d'envisager une résolution simplifiée ne prenant en compte que les chemins significatifs.

Sans pousser aussi loin l'analyse, l'examen de la plus grande des valeurs propres permet, dans le cas où elle est inférieure à 1, d'avoir recours à une solution itérative de l'équation du couplage (2) :

$$
\{\vec{w}\}=\sum_{n}[C]^{n}\left\{\vec{w}_{o}\right\}
$$

Cette résolution itérative peut être vue comme une succession d'échanges décroissants entre les deux systèmes. $\lambda<1$ apparaît donc comme un critère de couplage faible.

L'existence d'un tel mécanisme d'échanges entre systèmes couplés laisse entrevoir la possibilité d'une description énergétique de ces échanges.

\section{CONCLUSIONS}

Il a été démontré que le couplage entre deux systèmes pouvait être caractérisé par un nombre limité de données, éventuellement accessibles à la mesure : la valeur propre qui traduit la force du couplage et les vecteurs propres qui définissent les chemins de transmission. L'application de ces deux propriétés au cas des deux plaques couplées par un ressort a permis d'obtenir une solution analytique originale. L'exploitation de cette approche semble offrir de nombreuses possibilités, tant dans le domaine de la résolution numérique de problèmes couplés que dans les modèles énérgétiques du couplage.

\section{BIBLIOGRAPHIE}

[1] Gagliardini L., "Simulation numérique de la transmission acoustique par les parois simples et multiples", Thèse : Institut National des Sciences Appliquées de Lyon (1991) 352 p

[2] Gagliardini L., Roland J., Guyader J-L., "The use of a functionnal basis to calculate acoustic transmission between rooms", Journal of Sound and Vibrations 145 (3) (1991) 457-478

[3] Morand H. J-P., "A modal hybridization method for the reduction of dynamic models in the medium frequency range", Proceedings of the European Conference on New Advances in Computational Structural Mechanics (Ed. P. Ladeveze, O.C. Zienkiewicz, April 1991) pp. 1-18 